\title{
Myoclonic status epilepticus and cerebellar hypoplasia associated with a novel variant in the GRIA3 gene
}

\author{
Berardo Rinaldi ${ }^{1}$ Y Yu-Han Ge $\mathrm{G}^{2,3}$ - Elena Freri ${ }^{4}$ - Arianna Tucci ${ }^{5}$. Tiziana Granata ${ }^{4}$ Margherita Estienne ${ }^{4}$. \\ Jia-Hui Sun ${ }^{2,3} \cdot$ Bénédicte Gérard $^{6} \cdot$ Allan Bayat $^{7,8} \cdot$ Stephanie Efthymiou ${ }^{9} \cdot$ Cristina Gervasini $^{10} \cdot$ Yun Stone Shi $^{2,3}$. \\ Henry Houlden ${ }^{9} \cdot$ Paola Marchisio $^{1} \cdot$ Donatella Milani $^{1}$
}

Received: 3 March 2021 / Accepted: 1 August 2021 / Published online: 3 November 2021

(c) The Author(s) 2021, corrected publication 2021

\begin{abstract}
AMPA-type glutamate receptors (AMPARs) are postsynaptic ionotropic receptors which mediate fast excitatory currents. AMPARs have a heterotetrameric structure, variably composed by the four subunits GluA1-4 which are encoded by genes GRIA1-4. Increasing evidence support the role of pathogenic variants in GRIA1-4 genes as causative for syndromic intellectual disability (ID). We report an Italian pedigree where some male individuals share ID, seizures and facial dysmorphisms. The index subject was referred for severe ID, myoclonic seizures, cerebellar signs and short stature. Whole exome sequencing identified a novel variant in GRIA3, c.2360A > G, p.(Glu787Gly). The GRIA3 gene maps to chromosome Xq25 and the c.2360A $>\mathrm{G}$ variant was transmitted by his healthy mother. Subsequent analysis in the family showed a segregation pattern compatible with the causative role of this variant, further supported by preliminary functional insights. We provide a detailed description of the clinical evolution of the index subjects and stress the relevance of myoclonic seizures and cerebellar syndrome as cardinal features of his presentation.
\end{abstract}

Keywords GRIA3 · AMPARs · Glutamate $\cdot$ Myoclonic status epilepticus $\cdot$ Cerebellar hypoplasia

\section{Introduction}

The GRIA3 gene is located at $\mathrm{Xq} 25$ and encodes the AMPAtype glutamate receptor (AMPAR) subunit 3. AMPARs belong to ionotropic glutamate receptors and are responsible

Berardo Rinaldi and Yu-Han Ge are contributed equally to this work.

Arianna Tucci

a.tucci@qmul.ac.uk

Yun Stone Shi

yunshi@nju.edu.cn

1 Fondazione IRCCS Ca' Granda Ospedale Maggiore Policlinico, Milan, Italy

2 Ministry of Education Key Laboratory of Model Animal for Disease Study, Department of Neurology, Drum Tower Hospital, Medical School, Nanjing University, Nanjing, China

3 State Key Laboratory of Pharmaceutical Biotechnology, Model Animal Research Center, Institute for Brain Sciences, Chemistry and Biomedicine Innovation Center, Nanjing University, Nanjing, China

4 Department of Pediatric Neuroscience, Fondazione IRCCS Istituto Neurologico C. Besta, Milan, Italy
5 Clinical Pharmacology, William Harvey Research Institute, School of Medicine and Dentistry, Queen Mary University of London, London EC1M 6BQ, UK

6 Laboratoires de diagnostic génétique, Institut Medical d'Alsace, Hôpitaux Universitaire de Strasbourg, Strasbourg, France

7 Department for Genetics and Personalized Medicine, Danish Epilepsy Centre, Dianalund, Denmark

8 Institute for Regional Health Services Research, University of Southern Denmark, Odense, Denmark

9 Department of Neuromuscular disorders, UCL Queen Square Institute of Neurology, London, UK

10 Medical Genetics, Department of Health Sciences, Università degli Studi di Milano, Milan, Italy 
for fast excitatory postsynaptic currents. AMPARs are heterotetrameric receptors combining four different subunits, defined GluA1-4 and encoded by GRIA1-4 genes, respectively. GluA1-4 are differentially expressed during brain development and across the central nervous system $[1,2]$. AMPAR functional properties depend on the composition of the heterotetramer, their post-translational modifications and exposure rate onto the postsynaptic membrane [3-5]. Moreover, all the AMPAR genes express in vivo two different isoforms, generated by means of alternative splicing of exon 14 (flip/flop isoforms), which provides different kinetic behaviours and contributes to the functional richness of AMPARs [3, 6]. This tight regulation contributes to learning and memory processes via the synaptic plasticity [7-9].

Genetic variability in AMPAR genes has been associated with different neurological traits in humans such as sleep pattern [10], migraine [11,12] and psychoactive drug addiction [13, 14]. Currently, pathogenic variants in all the AMPAR genes have been reported in subjects presenting with syndromic intellectual disability. Deleterious variants currently described in GRIA1, GRIA2 and GRIA4, mapping respectively to chromosome 1,4 and 11 , are all de novo [15-17] whereas those in GRIA3 are mostly inherited from presumably healthy carrier mothers. Specifically for GRIA3, six unrelated pedigrees have been reported so far [18-20] and recurrent clinical features include intellectual disability, behavioural disorders and epilepsy. The employment of new sequencing techniques has identified further individuals, ascertained for early-onset epileptic encephalopathy, neurodevelopmental disorder, movement disorders and ID associated with abnormal sleep pattern [20-25]. Besides single nucleotide variants, the causative role of GRIA3 in XLID patients has been suggested also for Xq25 copy number variants $[18,26-30](\mathrm{CNVs})$ and for a balanced $\mathrm{t}(\mathrm{X} ; 12)$ (q24;q15) translocation disrupting the GRIA3 gene [31].

In the present study, we present a five-generation Italian pedigree with a novel GRIA3 variant co-segregating with a neurological phenotype in males.

\section{Methods}

\section{WES}

The proband has been evaluated with a long-term follow-up combining neurological and genetic expertise. The family expressed written consent for the publication. Genomic DNA was extracted from peripheral blood samples according to standard procedures of phenol chloroform extraction. WES on the trio was performed as described elsewhere [32] in Macrogen, Korea. Briefly, target enrichment was performed with $2 \mu \mathrm{g}$ genomic DNA using the SureSelectXT Human All Exon Kit version 6 (Agilent Technologies, Santa Clara, CA,
USA) to generate barcoded WES libraries. Libraries were sequenced on the HiSeqX platform (Illumina, San Diego, CA, USA) with $50 \times$ coverage. Quality assessment of the sequence reads was performed by generating QC statistics with FastQC. Our bioinformatics filtering strategy included screening for only exonic and donor/acceptor splicing variants. In accordance with the pedigree and phenotype, priority was given to rare variants $(<0.01 \%$ in public databases, including 1000 Genomes project, NHLBI Exome Variant Server, Complete Genomics 69 and Exome Aggregation Consortium [ExAC v0.2]) that were fitting a dominant (heterozygous/hemizygous) or a de novo model and/or variants in genes previously linked to intellectual disability and other neurological disorders. Variants of interest were confirmed with Sanger sequencing. Amplification reactions were performed with standard FastStart PCR reagents (Roche), on an ABI Veriti Thermal Cycler (Applied Biosystems). PCR products were purified using Exo-SAP (Exonuclease I and Shrimp Alkaline Phosphatase) and sequencing PCR was performed bi-directionally using BigDye Terminator Ready Reaction Mix kit version 3.1 (Applied Biosystems) and analyzed on an ABI 3730xl capillary sequencer. Electropherograms were generated on the Sequencher software to compare sequences of probands versus healthy controls. A similar approach was applied to conduct the segregation analysis in parents and in two male relatives. The significance of the identified variant was classified according to American College of Medical Genetics and Genomics (ACMG) criteria using Varsome tool [33].

\section{cDNA constructs}

cDNAs encoding human flop- and flip-type GluA3 (GluA3o and GluA3i, respectively) were subcloned into the NheI and XhoI restriction sites of the vector pCAGGS-IRES-EGFP. An HA epitope was inserted after the signal peptide (SP) of GluA3o, which read SP-GGGGS-HA-GGGGS-GluA3o. The GluA3o in this paper contains this HA epitope if not otherwise stated. Human GluA2 was subcloned into the vector pCAGGS-IRES-mCherry. Coexpression of GluA2 and GluA3 was identified by the fluorescence of EGFP and mCherry. GluA3i_E787G and GluA3o_E787G were made by overlapping PCR and confirmed by Sanger sequencing.

\section{HEK cells}

HEK293T cells were cultured in a $37{ }^{\circ} \mathrm{C}$ incubator supplied with 5\% CO2. Transfection was performed in 35-mm dishes using lipofectomine2000 reagents (Invitrogen). When coexpression was carried out, the ratio of GluA3 to GluA2 cDNA was 1:1. NBQX $(100 \mu \mathrm{M})$ was included in culture media to block AMPAR-induced cytotoxicity. Cells were dissociated with $0.05 \%$ trypsin and plated on coverslips pretreated with 
Fig. 1 Pedigree of the index family. Symptomatic individuals are shown in grey; the black arrow identifies the index subject; dashed circles identify possible female carriers
I

II

IV

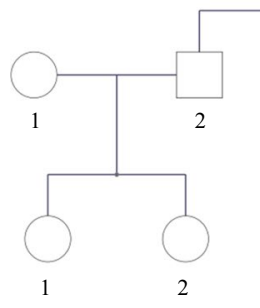

III

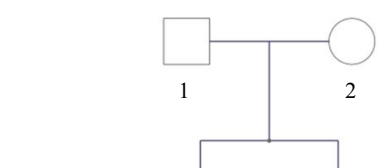

V
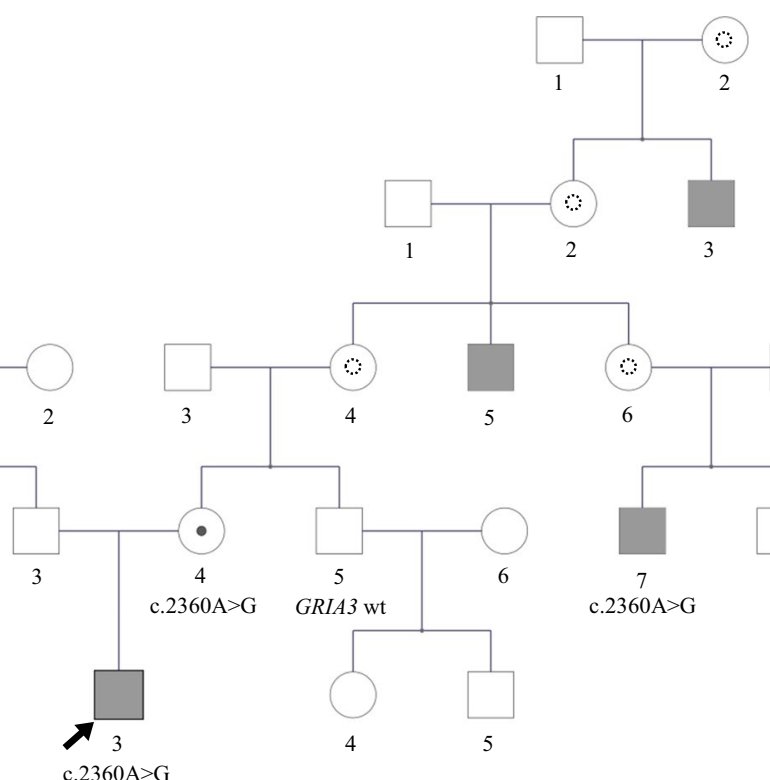

3
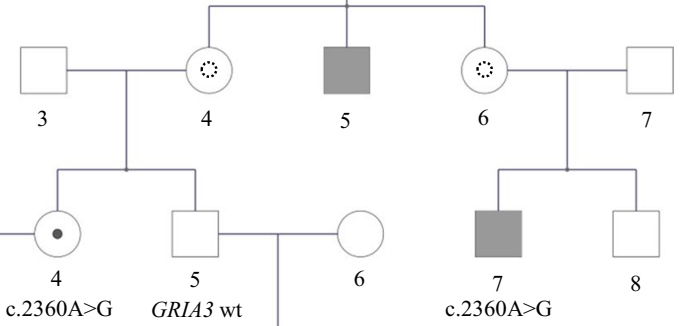

poly-D-lysine 24-h post transfection. Recording was performed $4 \mathrm{~h}$ after plating.

\section{Electrophysiology}

Receptor desensitization was recorded with whole-cell configuration of the transfected HEK 293 T cells. The external solution was as follows (in $\mathrm{mM}$ ): $140 \mathrm{NaCl}, 2.5 \mathrm{KCl}, 2$ $\mathrm{CaCl} 2,1 \mathrm{MgCl} 2,5$ glucose and 10 HEPES (pH 7.4). Patch pipettes (resistance 3 to $5 \mathrm{M} \Omega$ ) were filled with a solution containing the following (in $\mathrm{mM}$ ): $130 \mathrm{KF}, 33 \mathrm{KOH}$, $2 \mathrm{MgCl} 2,1 \mathrm{CaCl} 2,11$ EGTA and 10 HEPES (pH 7.4). Glutamate $(10 \mathrm{mM})$ diluted into the external solution was applied to lifted HEK cells with whole-cell configuration for $500 \mathrm{~ms}$ with a theta glass pipette mounted on a piezoelectric bimorph every $5 \mathrm{~s}$ [34]. Glutamate-induced currents were recorded with holding potential of $-70 \mathrm{mV}$. All the currents were collected with an Axoclamp 700B amplifier and Digidata 1440A (MolecularDevices, Sunnyvale, CA, USA), filtered at $2 \mathrm{kHz}$ and digitized at $100 \mathrm{kHz}$. The current data were analyzed using Clampfit software.

\section{Results}

The proband was referred at the age of 11 years for undiagnosed ID, drug-resistant epilepsy and ataxia. He was born to a healthy non consanguineous Italian couple. The family history revealed a 4th-degree relative with Down syndrome and four male relatives in the maternal lineage with variable association of ID, seizures and dysmorphisms (Fig. 1). The pregnancy was uneventful, except for few episodes of firsttrimester bleeding. Chorionic villus sampling was performed

due to the family history, showing a normal male karyotype. He was born at term and the neonatal weight was $2730 \mathrm{gr}$. During the first month, he underwent surgery for pyloric stenosis.

Psychomotor development was initially normal. Developmental delay and gait instability was firstly noticed at 15 months. Epilepsy onset occurred at 29 months with clonic seizures and myoclonic seizures with fall. Neurological examination was characterized by a cerebellar syndrome with ataxia, tremor and dysmetria. Interictal EEG revealed a poor organization of background activity, with bilateral high voltage activity and was dominated by epileptiform abnormalities prevalent on bilateral frontal regions; ictal discharges consisted of bilateral frontal or diffuse spike and wave. From the onset on, seizures quickly became very frequent and drug-resistant with recurrent myoclonic status epilepticus (Fig. 2). The patient was unsuccessfully treated with several therapeutic approaches: valproate, ethosuximide, methosuximide, clobazam, steroids such as ACTH and hydrocortisone, IGIV, bromide and clonazepam.

1,5-Tesla cerebral MRI described cerebellar vermis hypoplasia and a T1-weighted hyperintensity at the right frontal region, reviewed as a cortical dysplasia lesion by means of 3-Testla MRI (Fig. 3). Spectroscopy was normal.

His last physical evaluation was at the age of 18 years. Height was $161 \mathrm{~cm}\left(<3^{\circ}\right.$ percentile), weight was $47.5 \mathrm{~kg}$ $\left(<3^{\circ}\right.$ percentile) and $\mathrm{OFC}$ was $57 \mathrm{~cm}\left(90^{\circ}\right.$ percentile). $\mathrm{He}$ showed horizontal eyebrow, long eyelashes, bulbous nasal tip, underfolded helix, short philtrum, everted lower lip, and mild retrognathia (Fig. 4). Neurological examination revealed ataxia, severe intellectual disability with poor language and dysarthria, and behavioural disorder consisting in aggressive outbursts. From the age of 14, he became 


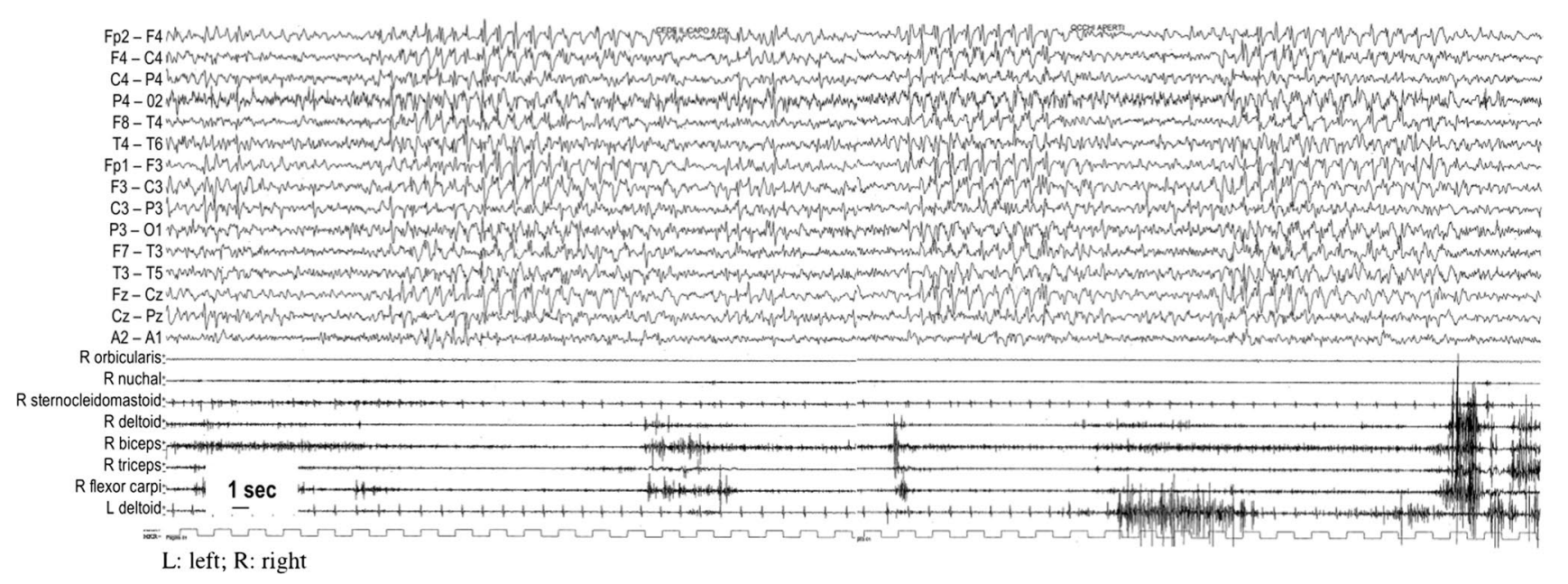

Fig. 2 EEG and electromyographic polygraphy during myoclonic status epilepticus (recorded at the age of 5 years, amplitude: 400 microVolt/cm)

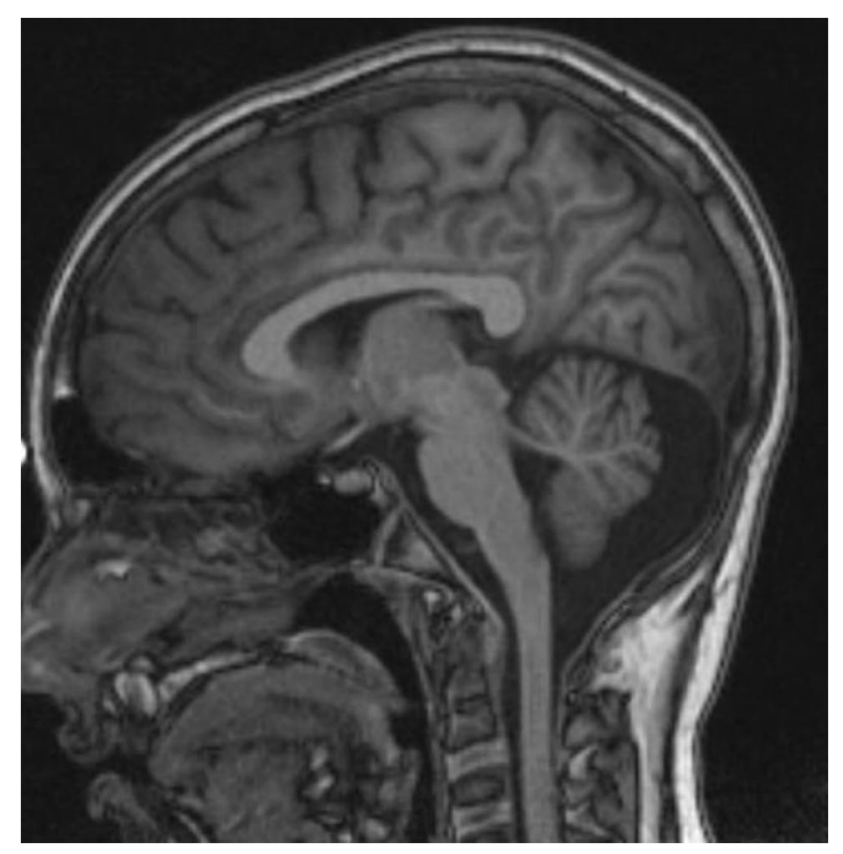

Fig. 3 Sagittal T1-weighted cerebral MRI scan showing vermian hypoplasia

seizure-free, being on a very low dosage of ethosuximide and clonazepam. His last EEG was performed at 17 years old and showed interictal diffuse epileptiform abnormalities during sleep.

In addition to 1st-tier metabolic screening, he underwent several genetic testing, all with normal results: FMRI trinucleotide expansion testing, chromosomal microarray (CMA), Sanger sequencing of $O P H N 1$ gene and targeted resequencing of SLC9A6, ARX, MECP2, CDKL5, FOXG1, UBE3A genes. WES identified two variants satisfying the filtering approach and no other class IV/V variants were found in his exome. The first one is the SLC16A2 gene variant NC_000023.10:g.73744270G $>$ A (NM_006517:c.652G > A, p.(Val218Ile)), which is present in Gnomad (AF 0.00001) and was interpreted as not causative also in view of the normality of the thyroid hormone profiles in the proband. The second one is the GRIA3 gene variant NC_000023.10:g.122599560A $>$ G (NM_000828:c.2360A > G, p.(Glu787Gly)), inherited from the asymptomatic mother. This variant maps to the exon 14 of the canonical transcript (NM_000828, flop isoform) whereas results in the intronic variant c. $2324+597 \mathrm{~A}>\mathrm{G}$ in the alternative isoform (NM_007325.5, flip isoform). When considering the canonical transcript, this variant was classified as likely pathogenic (PM1, PM2, PP1, PP2, PP3, PP4). In particular, this variant is absent in the GnomAD database and is predicted as deleterious by several bioinformatic pathogenicity scores (CADD score: 29.8; MutationTaster: disease causing; Sift4G: Damaging; PolyPhen-2: probably damaging). The Glu787 residue is conserved among species and in the primary sequence of the other AMPAR subunits as well (Fig. 5A-C). We conducted familial segregation analysis in one affected and in one unaffected relative of the maternal lineage (IV.7 and IV.5, respectively); as expected IV.7 carried the GRIA3 variant whereas IV.5 did not.

To better understand whether the variant induces biological changes, we tested its effect on glutamate-induced current. We cloned the human GluA3 subunit and tested its function by expression in HEK cells. Since the variant is located in exon 14, we thus cloned both the flop- and flip-GluA3 (Fig. 6A, GLUA3o and GLUA3i, respectively). The two amino acid sequences differ in nine residues, albeit glutamic acid 787 is present in both. When glutamate $(10 \mathrm{mM})$ was applied to HEK cells transfected with 
Fig. 4 Facial appearance of the index subject at the age of 11 (left) and 18 (right)
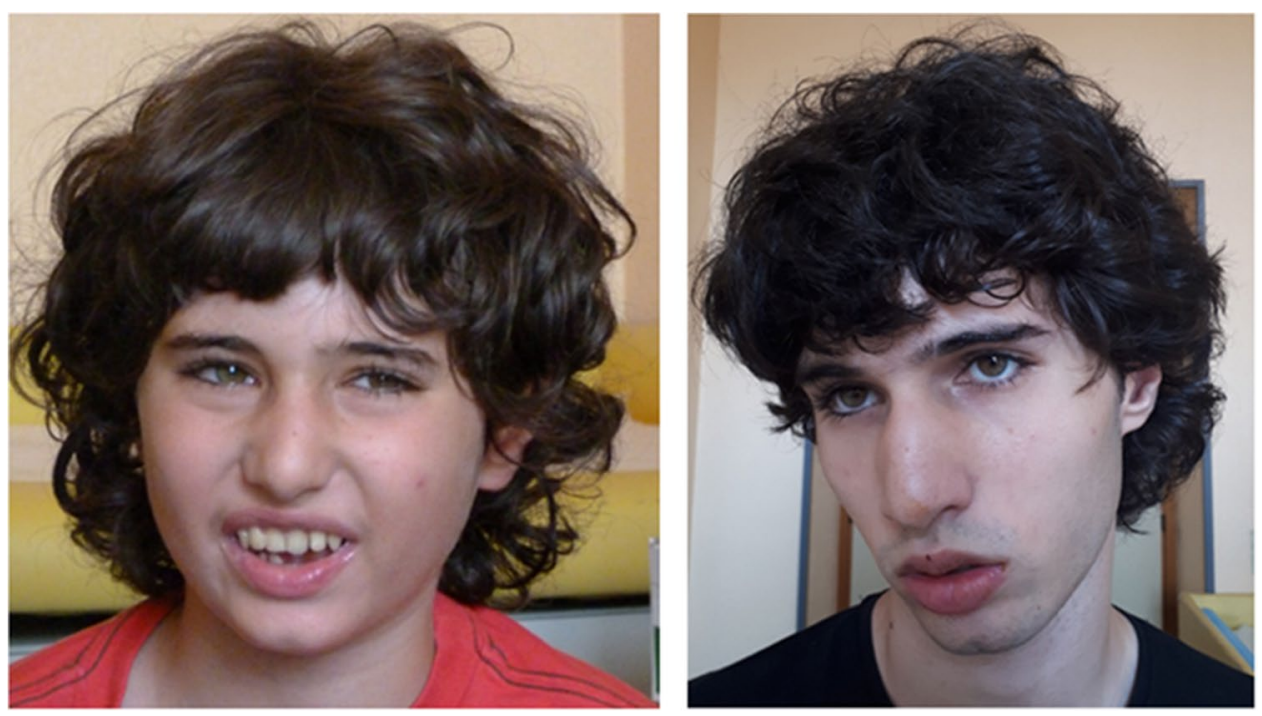

GluA3o and GluA3o_E787G, we observed the latter to produce no currents (Fig. 6B). In order to exclude that the $\mathrm{E} 787 \mathrm{G}$ mutant desensitizes too fast to be recorded, we applied the AMPAR desensitization blocker, trichloromethiazide (TCM, $100 \mu \mathrm{M}$ ), together with glutamate and still measured no currents (Fig. 6C). Also in the case of the flip isoform, no glutamate-induced currents were recorded in GluA3i-E787G transfected HEK cells, whilst GluA3i induced significant currents (Fig. 6D). Since GluA3 forms heteromeric $\mathrm{Ca} 2+$-impermeable AMPARs with GluA2 in the brain, we thus coexpressed GluA3i with GluA2i(R) and again we did not observe significant current in GluA2/ A3i_E787G (Fig. 6E). Taken together these data support the pathogenic role of the E787G substitution and the functional importance of the variant g.122599560A > G in both the flip and flop isoform.

\section{Discussion}

Neurodevelopmental disorders (NDD) and epilepsy often coexist in individuals referred for genetic investigations. More than distinct comorbidities, this reflects the variable clinical outcome of mutated genes with critical roles in the central nervous system [35]. As an example, ion channel dysfunction has been associated with a wide range of neurological conditions, either isolated or syndromic. Besides epilepsy and epileptic encephalopathies, the clinical spectrum of channelopathies also includes ataxia, movement disorders, ID, autism spectrum disorders (ASD), migraine, sleep disorders, cardiac arrhythmias and sudden unexpected death in epilepsy (SUDEP) [36]. In this context, few recent studies identified causative variants in genes encoding AMPAR subunits for individuals with NDD associated with epilepsy [15-17, 19, 24, 25].

Here, we report a novel GRIA3 variant, c.2360A > G p.(Glu787Gly), co-segregating with a syndromic NDD in an Italian pedigree. The neurological phenotype of our proband mostly concerns three domains: developmental delay (DD) evolving in severe ID, cerebellar signs due to vermian hypoplasia and clonic/myoclonic seizures even configuring myoclonic status epilepticus. Moreover, he showed short stature, low weight, relative macrocephaly and facial dysmorphisms. This clinical presentation recalls what is already known for pathogenic variants in GRIA3, i.e. moderate-severe ID, seizures and myoclonic jerks, short stature with macrocephaly [18, 19, 24]. We show that the epileptic phenotype associated to GRIA3 haploinsufficiency may manifest with refractory myoclonic status epilepticus, though the proband demonstrated an overall ameliorative evolution, becoming seizure-free from the age of 14. As to autism spectrum disorders and behavioural issues, also described in individuals with GRIA3 deleterious variants, our patient did not express autistic traits whereas he occasionally showed oppositional conduct and aggressive outbursts. Cerebral MRI of our proband highlighted cerebellar vermis hypoplasia and a focal cortical dysplasia. Though robust neuroimaging data on male individuals with impairment of GRIA3 have not been gathered yet, two patients with $\mathrm{Xq} 25$ duplications involving GRIA3 revealed a thin corpus callosum and moderate superior vermian atrophy $[26,30]$. On the other hand, their duplication includes other dosage-sensitive gene as STAG2, whose involvement may lead to corpus callosum abnormalities as well. With specific regard to the abnormal sleep pattern previously described in association with the p.(Ala653Thr) 
a)

\begin{tabular}{|c|c|c|c|c|c|c|c|c|c|c|c|c|c|c|c|c|c|c|c|c|c|c|c|c|c|c|}
\hline \multicolumn{4}{|c|}{$776-806$ (31r shown) } & \multicolumn{3}{|c|}{$\diamond \Leftrightarrow$} & . & & & & & & + & T & & & & & & & & & & & \multicolumn{2}{|c|}{ Downlosd $\mid$ \& Tools $=\mid \hat{e} ?}$. \\
\hline Sequence ID & & Start & $\stackrel{776}{+}$ & & 778 & & 780 & & 782 & & 784 & & $\underset{786}{+}$ & & 788 & & $\underset{790}{1}$ & & $\stackrel{792}{+}$ & & $\underset{194}{1}$ & & $\stackrel{796}{1}$ & & End & Organism \\
\hline NP 015564.5 & 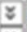 & 1 & $\mathrm{~T}$ & $\mathbf{P}$ & v & N & L. & A & $\mathrm{v}$ & L & $\mathbf{K}$ & L & $\mathbf{s}$ & E & $Q$ & G & I & L & D & $\mathbf{K}$ & L & $\mathbf{K}$ & N & $\mathrm{K}$ & 894 & Homo saplens \\
\hline Xe 014983615.1 & $\bar{z}$ & 1 & $\mathrm{~T}$ & $\mathbf{P}$ & v & N & L. & A & v & $\mathbf{L}$ & $\mathbf{K}$ & L & $\mathbf{s}$ & $\mathbf{E}$ & 2 & G & $\mathbf{I}$ & L & D & $\mathbf{K}$ & L & $\mathbf{K}$ & N & $\mathrm{K}$ & 894 & Macaca mulatta \\
\hline NP osses 82.3 & $\bar{z}$ & 1 & $\mathrm{~T}$ & $\mathbf{P}$ & $\mathbf{v}$ & N & L & $\mathbf{A}$ & v & $\mathbf{L}$ & $\mathbf{K}$ & L & $\mathbf{s}$ & $\mathbf{E}$ & $Q$ & G & I & L. & D & $\mathbf{K}$ & L & $\mathbf{K}$ & $\mathbf{N}$ & $\mathrm{K}$ & 888 & Mus musculus \\
\hline$x 0852016.1$ & $\bar{x}$ & 1 & $\mathrm{~T}$ & $\mathbf{P}$ & v & N & L & $\mathbf{A}$ & V & L & $\mathbf{K}$ & L & $\mathbf{s}$ & $\mathbf{E}$ & $Q$ & G & I & L & D & $\mathbf{K}$ & L & $\mathbf{K}$ & $\mathbf{N}$ & K & 894 & Canis luous familiaris \\
\hline XP 003414810.1 & $\frac{8}{n}$ & 1 & $T$ & $\mathbf{P}$ & $\mathbf{v}$ & $N$ & L & A & $\mathrm{v}$ & L & $\mathbf{K}$ & L & s & $\mathbf{z}$ & $Q$ & $G$ & I & L & D & K & L & $\mathbf{K}$ & N & $\mathrm{K}$ & 894 & Loxodonta africana \\
\hline NP 990546.2 & $y$ & 1 & $N$ & A & $\mathrm{v}$ & $N$ & L & A & $\mathrm{v}$ & L & $\mathbf{x}$ & L & $N$ & $\mathbf{z}$ & $Q$ & G & $L$ & L & D & $\mathrm{K}$ & $L$ & $\mathbf{K}$ & N & $\mathrm{K}$ & 888 & Gallus oallus \\
\hline NP 001106494.1 & $\bar{z}$ & 1 & $T$ & $\mathbf{P}$ & v & $N$ & L. & $\mathbf{A}$ & V & $\mathbf{L}$ & $\mathbf{K}$ & L & s & $\mathbf{z}$ & $Q$ & G & I & I. & D & $\mathrm{K}$ & L & $\mathbf{K}$ & $\mathbf{N}$ & $\mathbf{K}$ & 887 & Xenoous trooicalis \\
\hline NP 938174.1 & 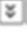 & 1 & N & A & $\mathbf{v}$ & N & L & A & v & L & $\mathbf{K}$ & L & $N$ & $\mathbf{E}$ & $Q$ & G & L & L & D & $\mathbf{K}$ & L & $\mathbf{K}$ & N & K & 883 & Danio rerio \\
\hline
\end{tabular}

P42261 GRIA1_HUMAN 758 NPVNLAVLKLNEQGLLDKLKNKWW

b) P42262 GRIA2 ${ }^{-}$HUMAN 765 NAVNLAVLKLNEQGLLDKLKNKWW

P42263 GRIA3 ${ }^{-}$HUMAN 776 NAVNLAVLKLNEQGLLDKLKNKWW

P48058 GRIA4 HUMAN 766 TPVNLAVLKLSEAGVLDKLKNKWW

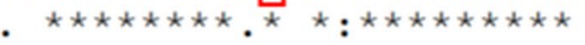

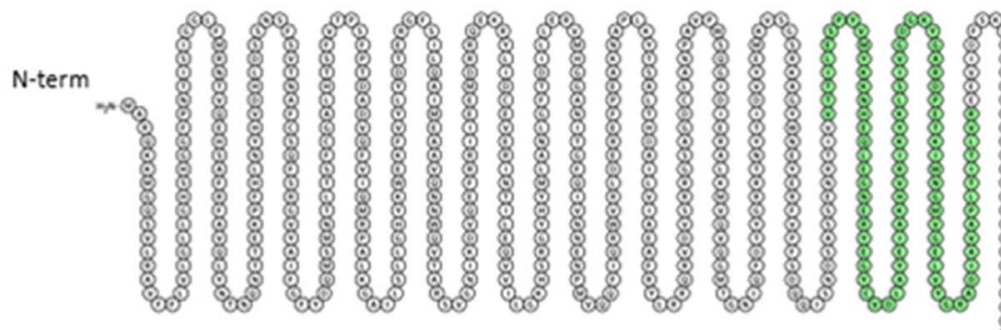

c)
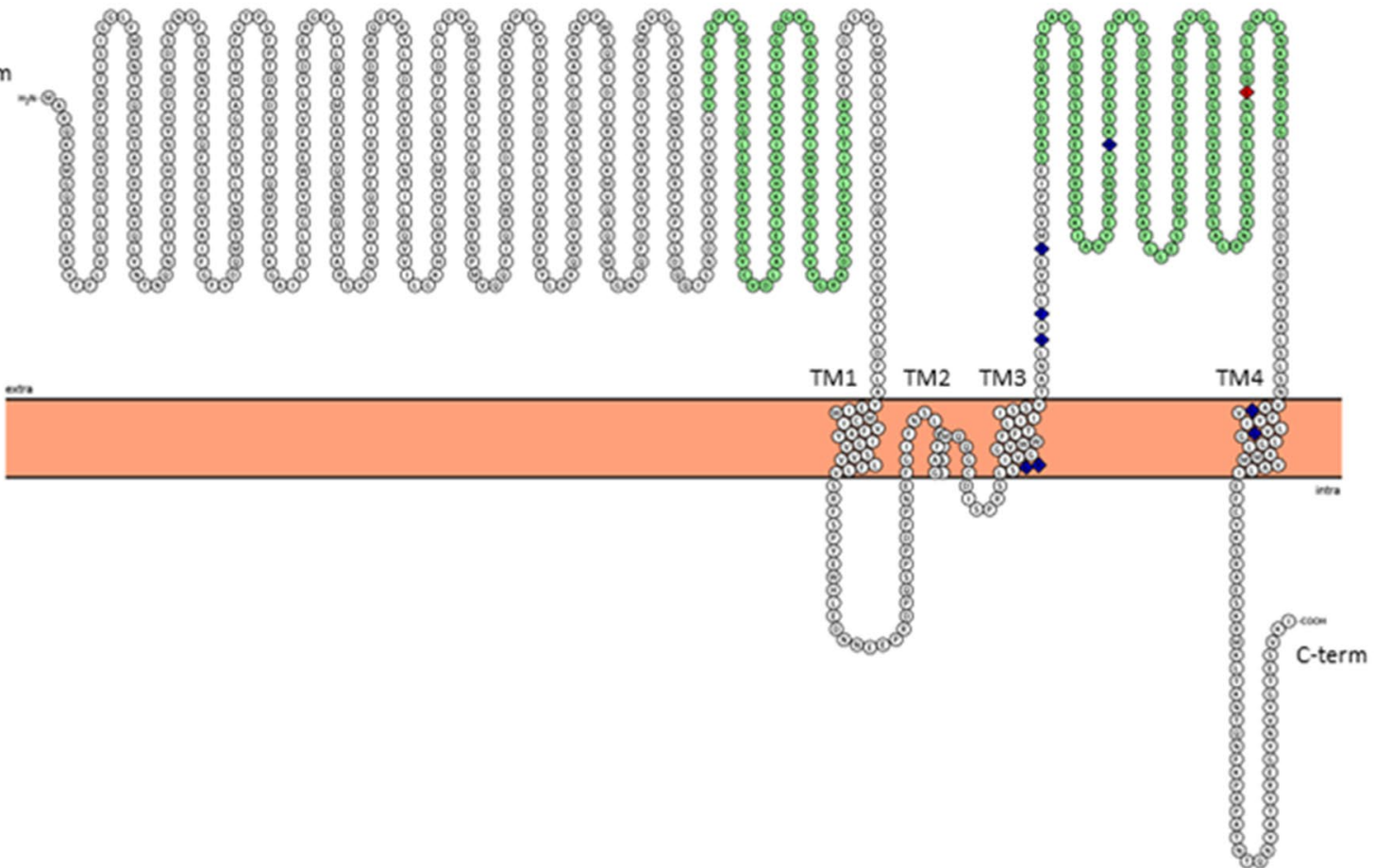

Fig. 5 Conservation and localization of the Glu787 residue. (a) Phylogenetic conservation among different species; (b) conservation among the primary structure of human GluA1-4 subunits; (c) graphical representation of the GluA3 subunit, published mutated residues

variant in GRIA3 [22], any alteration of the circadian cycle was reported for our proband. Comparing the facial gestalt of our proband with others already published, few traits look recurrent, as for the hypotonic facies, malar flattening, short philtrum, thick vermillion of the lips with eversion of the lower lip $[19,30]$.

The identified p.(Glu787Gly) variant is located within the ligand-binding domain (LBD) of the receptor where glutamate binds and triggers the channel opening. Testing the functional effects of missense variants on synaptic receptors is puzzling as the amino acid change may lead to different and possibly discordant results depending on the state of
$18,19,20,22,23,25$ are shown in blue, the Glu787 residue in red. The green residues constitute the ligand-binding domain (LBD). Figure $5 \mathrm{a}, 5 \mathrm{~b}$ and $5 \mathrm{c}$ were prepared with the help of COBALT (NCBI), UniProt and Protter, respectively (please refer to Web Resources)

the channel (active, inactive, desensitized). In our case, the scenario is further complicated by the location of the variant, being unknown a priori whether the variant is expressed (flop isoform) and to which extent.

Therefore, in this study, we started to characterize the GRIA3 variant c.2360A $>$ G p.(Glu787Gly). The absence of current of the mutant receptor in any of the tested settings may suggest that the g.122599560A $>\mathrm{G}$ variant leads to a loss of function of the subunit in both isoforms. If this result may be conceivable for the flop isoform, the mechanisms responsible for the impairment of the flip isoform remain to be determined. Further studies are then needed to expand our findings and 
Fig. 6 Glutamate-induced currents in wild-type GluA3 and in the E787G mutant. (A) The flip and flop of GLUA3. (B) Glutamate-induced currents in GLUA3o and GLUA3o_E787G. Left panel, sample traces. Right panel, bar graph showing the glutamate-induced current amplitudes. GLUA3o, $242.6 \pm 44.5 \mathrm{pA}, n=10$; GLUA3o_E787G, $2.9 \pm 0.3$ $\mathrm{pA}, n=6$. (C) Glutamateinduced currents in GLUA3o and GLUA3o_E787G in the presence of TMC. GLUA3o, $436.2 \pm 56.7 \mathrm{pA}, n=11$; GLUA3o_E787G, $2.6 \pm 0.4$ $\mathrm{pA}, n=9$. (D) Glutamateinduced currents in GLUA3i and GLUA3i-E787G. GLUA3i, $528.7 \pm 67.7 \mathrm{pA}, n=12$; GLUA3i_E787G, $3.6 \pm 0.4$, $n=3$. (E) Glutamate-induced currents in GLUA3i and GLUA3i_E787G with the coexpression of GLUA2i. GLUA2/ A3i, 477.1 $\pm 107.2 \mathrm{pA}, n=11$; GLUA2/A3i_E787G, $7.8 \pm 1.2$, $n=3$. Data are presented as means \pm SEM. $* * * p<0.001$, unpaired $t$-test
A

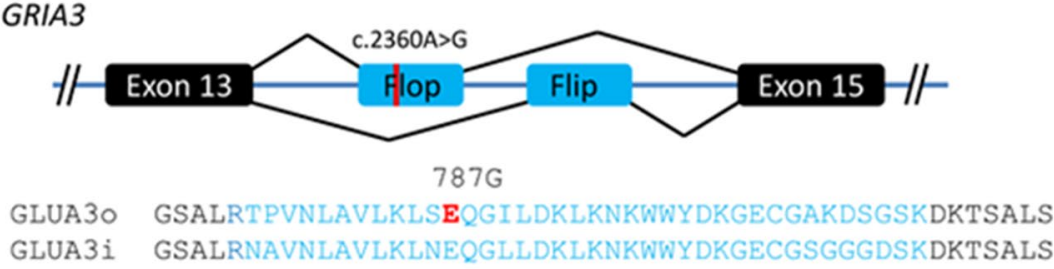

B
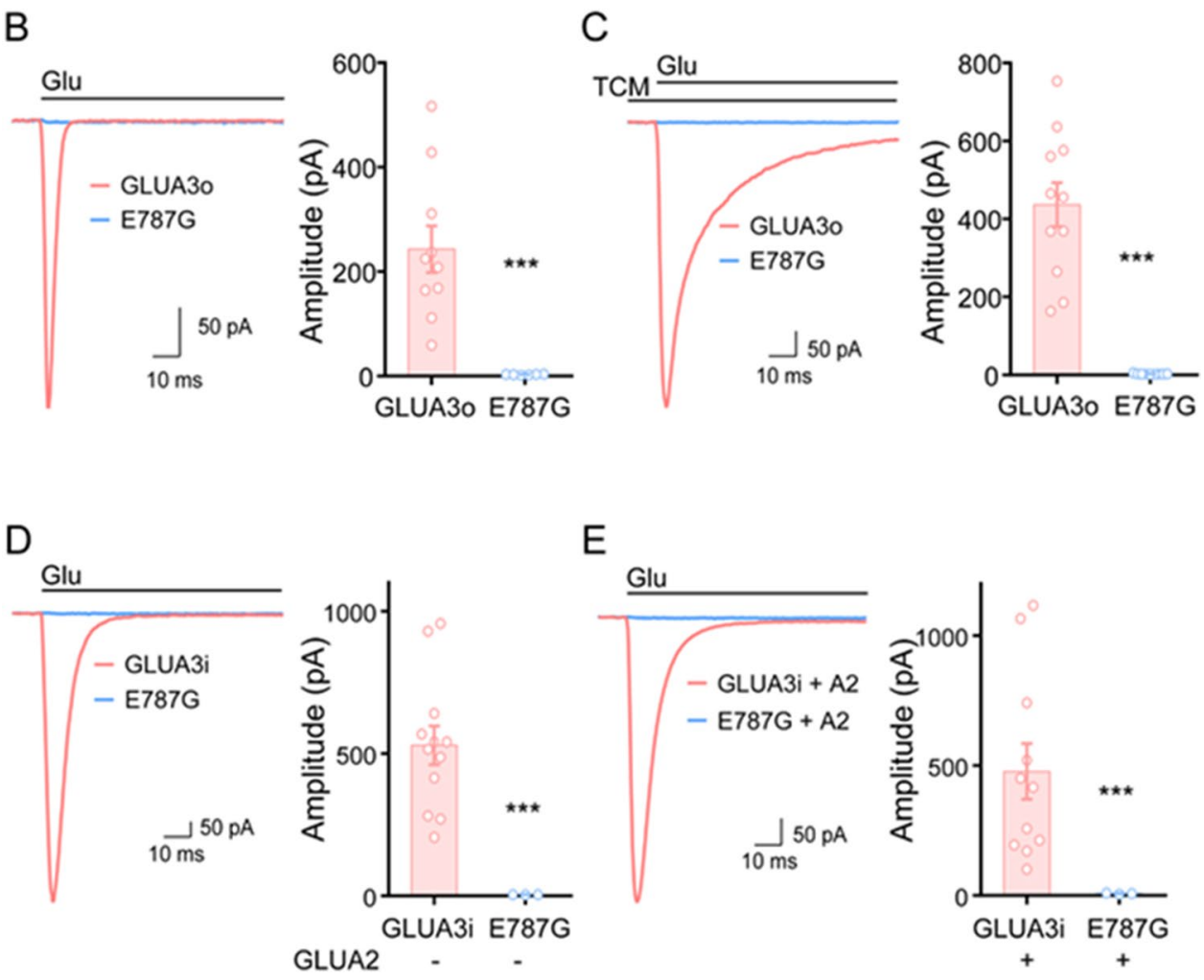

E

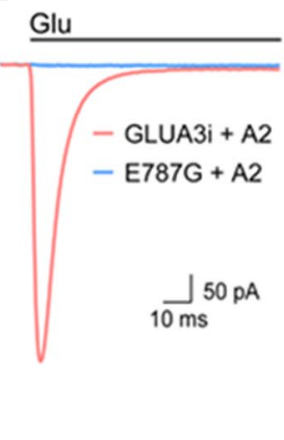

provide a deeper understanding. A different pathogenic variant affecting the same amino acid residue was recently described in another Italian male child [24]. Interestingly, the two patients experienced status epilepticus and myoclonic seizures but with differences in epileptic severity and neuroimaging findings. Moreover, for the male patient reported by Trivisano et al., a targeted trial with a selective non-competitive AMPA receptor antagonist (Perampanel) proved to be ineffective, as expected in case of loss of function of the receptor (Fig. 6).

Because of the heterotetrameric structure of AMPARs, pathogenic variants in each of GRIAl-4 genes might exert dominant effects on other subunits of AMPARs with convergent effects on the resulting phenotypes, as also suggested by functional studies [22]. Our proband shares some of the clinical features already associated with other GRIA genes. This includes early normal psychomotor development, speech delay more pronounced than the motor impairment, cerebellar MRI findings and neurological signs. Based on published data, the most overlapping pattern would appear with cases with pathogenic variants in GRIA2 and, indeed, GluA3 co-assembles mostly with GluA2 to generate AMPARs [37]. On the other hand, the corresponding residue in the GluA2 subunit has already been found mutated (p.(Glu776Asp)) in an individual with a slightly different phenotype, consisting of DD/ID, ASD, insomnia, normal brain MRI and a single episode of generalized tonic seizure [16]. Therefore, the small numbers of patients and the wide range of functional effects produced by missense variants make stringent genotype-phenotype correlation for GRIA genes still uncertain.

\section{Web resources}

CADD [38] (https://cadd.gs.washington.edu/)

COBALT (https://www.ncbi.nlm.nih.gov/tools/cobalt/re_ cobalt.cgi)

FastQC (https://www.bioinformatics.babraham.ac.uk/ projects/fastqc/)

GnomAD [39] (https://gnomad.broadinstitute.org/)

MutationTaster [40] (http://www.mutationtaster.org/) 
PolyPhen-2 [41] (http://genetics.bwh.harvard.edu/pph2/) Protter [42] (http://wlab.ethz.ch/protter/)

Sift4G [43] (https://sift.bii.a-star.edu.sg/sift4g/)

UniProt [44] (https://www.uniprot.org/)

VarSome [33] (https://varsome.com/)

Acknowledgements The authors want to thank the family for their collaboration, Prof. Anders Skov Kristensen (University of Copenhagen) for his critical review of the paper, Dr. Laura Canafoglia (Neurophysiopathology team at Fondazione IRCCS Istituto Neurologico Carlo Besta) for Fig. 2. Fondazione IRCSS Ca' Granda Ospedale Maggiore Policlinico is a member of the ERN-ITHACA network (https:// ern-ithaca.eu/). Fondazione IRCCS Istituto Neurologico C. Besta is a member of the ERN-EpiCARE network (https://epi-care.eu/).

Author contributions B.R., A.T., D.M., and Y.S.S. designed the study; B.R. reviewed the literature and drafted the initial manuscript; B.R., P.M. and D.M. reviewed the clinical data and the manuscript; Y.H.G, J.H.S and Y.S.S performed and interpreted the functional studies and drafted the manuscript; E.F., T.G. and M.E. reviewed the neurological data and drafted the manuscript; A.T., S.E., C.G. and H.H. performed the WES or the segregation analysis and reviewed the manuscript; B.G. and A.B. reviewed the genotype-phenotype correlation and the manuscript.

Funding This work is supported by grants from National Key R \& D Program of China (2019YFA0801603 to Y.S.S.), the National Natural Science Foundation of China (91849112 to Y.S.S), the Natural Science Foundation of Jiangsu Province (BE2019707 to Y.S.S.) and Fundamental Research Funds for the Central Universities (0903-14380029 to Y.S.S.). The funders had no role in study design, data collection and analysis, decision to publish, or preparation of the manuscript.

Open Access This article is licensed under a Creative Commons Attribution 4.0 International License, which permits use, sharing, adaptation, distribution and reproduction in any medium or format, as long as you give appropriate credit to the original author(s) and the source, provide a link to the Creative Commons licence, and indicate if changes were made. The images or other third party material in this article are included in the article's Creative Commons licence, unless indicated otherwise in a credit line to the material. If material is not included in the article's Creative Commons licence and your intended use is not permitted by statutory regulation or exceeds the permitted use, you will need to obtain permission directly from the copyright holder. To view a copy of this licence, visit http://creativecommons.org/licenses/by/4.0/.

\section{References}

1. Beneyto M, Meador-Woodruff JH (2004) Expression of transcripts encoding AMPA receptor subunits and associated postsynaptic proteins in the macaque brain. J Comp Neurol 468(4):530-554. https://doi.org/10.1002/cne.10981

2. Pickard L, Noel J, Henley JM, Collingridge GL, Molnar E (2000) Developmental changes in synaptic AMPA and NMDA receptor distribution and AMPA receptor subunit composition in living hippocampal neurons. J Neurosci 20(21):7922-7931. https://doi. org/10.1523/jneurosci.20-21-07922.2000

3. Sommer B, Keinänen K, Verdoorn TA, Wisden W, Burnashev N, Herb A et al (1990) Flip and flop: a cell-specific functional switch in glutamate-operated channels of the CNS. Science 249(4976):1580-1585. https://doi.org/10.1126/science.1699275
4. Greger IH, Khatri L, Kong X, Ziff EB (2003) AMPA receptor tetramerization is mediated by Q/R editing. Neuron 40(4):763774. https://doi.org/10.1016/S0896-6273(03)00668-8

5. Petrini EM, Lu J, Cognet L, Lounis B, Ehlers MD, Choquet D (2009) Endocytic trafficking and recycling maintain a pool of mobile surface AMPA receptors required for synaptic potentiation. Neuron 63(1):92-105. https://doi.org/10.1016/j.neuron.2009. 05.025

6. Pei W, Huang Z, Niu L (2007) GluR3 flip and flop: differences in channel opening kinetics. Biochemistry 46(7):2027-2036. https:// doi.org/10.1021/bi062213s

7. Liu SJ, Zukin RS (2007) Ca2+-permeable AMPA receptors in synaptic plasticity and neuronal death. Trends Neurosci 30(3):126-134. https://doi.org/10.1016/J.TINS.2007.01.006

8. Cull-Candy S, Kelly L, Farrant M (2006) Regulation of Ca2+permeable AMPA receptors: synaptic plasticity and beyond. Curr Opin Neurobiol 16(3):288-297. https://doi.org/10.1016/J.CONB. 2006.05.012

9. Renner MC., Albers EH., Gutierrez-Castellanos N., Reinders NR., Van Huijstee AN., Xiong H., et al. Synaptic plasticity through activation of GluA3-containing AMPA-receptors 2017. Doi: https://doi.org/10.7554/eLife.25462.001

10. Utge S, Kronholm E, Partonen T, Soronen P, Ollila HM, Loukola A et al (2011) Shared genetic background for regulation of mood and sleep: association of GRIA3 with sleep duration in healthy Finnish women. Sleep 34(10):1309-1316. https://doi.org/10.5665/ sleep. 1268

11. Formicola D, Aloia A, Sampaolo S, Farina O, Diodato D, Griffiths LR et al (2010) Common variants in the regulative regions of GRIA1 and GRIA3 receptor genes are associated with migraine susceptibility. BMC Med Genet 11(1):103. https://doi.org/10. 1186/1471-2350-11-103

12. Maher BH, Lea RA, Follett J, Cox HC, Fernandez F, Esposito $\mathrm{T}$ et al (2013) Association of a GRIA3 gene polymorphism with migraine in an Australian case-control cohort. Headache 53(8):1245-1249. https://doi.org/10.1111/head.12151

13. Iamjan SA, Thanoi S, Watiktinkorn P, Reynolds GP, NudmamudThanoi S (2018) Genetic variation of GRIA3 gene is associated with vulnerability to methamphetamine dependence and its associated psychosis. J Psychopharmacol 32(3):309-315. https://doi. org/10.1177/0269881117750153

14. Acosta G, Freidman DP, Grant KA, Hemby SE (2012) Alternative splicing of AMPA subunits in prefrontal cortical fields of cynomolgus monkeys following chronic ethanol self-administration. Front Psychiatry 2:72. https://doi.org/10.3389/fpsyt.2011.00072

15. Martin S, Chamberlin A, Shinde DN, Hempel M, Strom TM, Schreiber A et al (2017) De novo variants in GRIA4 lead to intellectual disability with or without seizures and gait abnormalities. Am J Hum Genet 101(6):1013-1020. https://doi.org/10.1016/j. ajhg.2017.11.004

16. Salpietro V, Dixon CL, Guo H, Bello OD, Vandrovcova J, Efthymiou $\mathrm{S}$ et al (2019) AMPA receptor GluA2 subunit defects are a cause of neurodevelopmental disorders. Nat Commun 10(1):3094. https://doi.org/10.1038/s41467-019-10910-w

17. Geisheker MR., Heymann G., Wang T., Coe BP., Turner TN., Stessman HAF., et al. Hotspots of missense mutation identify neurodevelopmental disorder genes and functional domains. Nat Neurosci 2017;20(8):1043-51. Doi: https://doi.org/10.1038/nn. 4589

18. Wu Y, Arai AC, Rumbaugh G, Srivastava AK, Turner G, Hayashi $\mathrm{T}$ et al (2007) Mutations in ionotropic AMPA receptor 3 alter channel properties and are associated with moderate cognitive impairment in humans. Proc Natl Acad Sci U S A 104(46):1816318168. https://doi.org/10.1073/pnas.0708699104

19. Philips AK, Sirén A, Avela K, Somer M, Peippo M, Ahvenainen $M$ et al (2014) X-exome sequencing in Finnish families with 
intellectual disability - four novel mutations and two novel syndromic phenotypes. Orphanet J Rare Dis 9(1):49. https://doi.org/ 10.1186/1750-1172-9-49

20. Piard J., Béreau M., XiangWei W., Wirth T., Amsallem D., Buisson L., et al. The GRIA3 c. $2477 \mathrm{G}>$ A variant causes an exaggerated startle reflex, chorea, and multifocal myoclonus. Mov Disord 2020;35(7):1224-32. Doi: https://doi.org/10.1002/mds.28058

21. Allen NM., Conroy J., Shahwan A., Lynch B., Correa RG., Pena SDJ., et al. Unexplained early onset epileptic encephalopathy: exome screening and phenotype expansion. Epilepsia 2016;57(1):e12-7. Doi: https://doi.org/10.1111/epi.13250

22. Davies B, Brown LA, Cais O, Watson J, Clayton AJ, Chang VT et al (2017) A point mutation in the ion conduction pore of AMPA receptor GRIA3 causes dramatically perturbed sleep patterns as well as intellectual disability. Hum Mol Genet 26(20):3869-3882. https://doi.org/10.1093/hmg/ddx270

23. Chérot E, Keren B, Dubourg C, Carré W, Fradin M, Lavillaureix A et al (2018) Using medical exome sequencing to identify the causes of neurodevelopmental disorders: experience of 2 clinical units and 216 patients. Clin Genet 93(3):567-576. https:// doi.org/10.1111/cge. 13102

24. Trivisano M, Santarone ME, Micalizzi A, Ferretti A, Dentici ML, Novelli A et al (2020) GRIA3 missense mutation is cause of an $\mathrm{x}$-linked developmental and epileptic encephalopathy. Seizure 82:1-6. https://doi.org/10.1016/j.seizure.2020.08.032

25. Sun J-H., Chen J., Ayala Valenzuela FE., Brown C., MasserFrye D., Jones M., et al. X-linked neonatal-onset epileptic encephalopathy associated with a gain-of-function variant p.R660T in GRIA3. PLOS Genet 2021;17(6):e1009608. Doi: https://doi.org/10.1371/journal.pgen.1009608

26. Jacquemont ML, Sanlaville D, Redon R, Raoul O, CormierDaire V, Lyonnet S et al (2006) Array-based comparative genomic hybridisation identifies high frequency of cryptic chromosomal rearrangements in patients with syndromic autism spectrum disorders. J Med Genet 43(11):843-849. https://doi. org/10.1136/jmg.2006.043166

27. Chiyonobu T, Hayashi S, Kobayashi K, Morimoto M, Miyanomae Y, Nishimura A et al (2007) Partial tandem duplication of GRIA3 in a male with mental retardation. Am J Med Genet Part A 143(13):1448-1455. https://doi.org/10.1002/ajmg.a.31798

28. Guilmatre A, Dubourg C, Mosca AL, Legallic S, Goldenberg A, Drouin-Garraud V et al (2009) Recurrent rearrangements in synaptic and neurodevelopmental genes and shared biologic pathways in schizophrenia, autism, and mental retardation. Arch Gen Psychiatry 66(9):947-956. https://doi.org/10.1001/archgenpsychiatry.2009.80

29. Bonnet C, Leheup B, Béri M, Philippe C, Grégoire MJ, Jonveaux P (2009) Aberrant GRIA3 transcripts with multi-exon duplications in a family with X-linked mental retardation. Am J Med Genet Part A 149(6):1280-1289. https://doi.org/10.1002/ajmg.a.32858

30. Philippe A, Malan V, Jacquemont M-L, Boddaert N, Bonnefont J-P, Odent $\mathrm{S}$ et al (2013) Xq25 duplications encompassing GRIA 3 and STAG2 genes in two families convey recognizable X-linked intellectual disability with distinctive facial appearance. Am J Med Genet Part A 161(6):1370-1375. https://doi.org/10.1002/ ajmg.a. 35307

31. Gécz J, Barnett S, Liu J, Hollway G, Donnelly A, Eyre H et al (1999) Characterization of the human glutamate receptor subunit 3 gene (GRIA3), a candidate for bipolar disorder and nonspecific
X-linked mental retardation. Genomics 62(3):356-368. https:// doi.org/10.1006/geno.1999.6032

32. Mencacci NE, Kamsteeg EJ, Nakashima K, R'Bibo L, Lynch DS, Balint B et al (2016) De novo mutations in PDE10A cause childhood-onset chorea with bilateral striatal lesions. Am J Hum Genet 98(4):763-771. https://doi.org/10.1016/j.ajhg.2016.02.015

33. Kopanos C, Tsiolkas V, Kouris A, Chapple CE, Albarca AM, Meyer R et al (2019) VarSome: the human genomic variant search engine. Bioinformatics 35(11):1978-1980. https://doi.org/10. 1093/bioinformatics/bty897

34. Li YJ, Duan GF, Sun JH, Wu D, Ye C, Zang YY et al (2019) Neto proteins regulate gating of the kainate-type glutamate receptor GluK2 through two binding sites. J Biol Chem 294(47):1788917902. https://doi.org/10.1074/jbc.RA119.008631

35. Heyne HO, Singh T, Stamberger H, Abou JR, Caglayan H, Craiu $D$ et al (2018) De novo variants in neurodevelopmental disorders with epilepsy. Nat Genet 50(7):1048-1053. https://doi.org/10. 1038/s41588-018-0143-7

36. Bartolini E, Campostrini R, Kiferle L, Pradella S, Rosati E, Chinthapalli K et al (2019) Epilepsy and brain channelopathies from infancy to adulthood. Neurol Sci. https://doi.org/10.1007/ s10072-019-04190-x

37. Wenthold RJ, Petralia RS, Blahos J, Niedzielski AS (1996) Evidence for multiple AMPA receptor complexes in hippocampal CA1/CA2 neurons. J Neurosci 16(6):1982-1989. https://doi.org/ 10.1523/jneurosci.16-06-01982.1996

38. Kircher M, Witten DM, Jain P, O'Roak BJ, Cooper GM, Shendure J (2014) A general framework for estimating the relative pathogenicity of human genetic variants. Nat Genet 46(3):310-315. https://doi.org/10.1038/ng.2892

39. Karczewski KJ, Francioli LC, Tiao G, Cummings BB, Alföldi J, Wang Q et al (2020) The mutational constraint spectrum quantified from variation in 141,456 humans. Nature 581(7809):434443. https://doi.org/10.1038/s41586-020-2308-7

40. Schwarz JM, Cooper DN, Schuelke M, Seelow D (2014) Mutationtaster2: mutation prediction for the deep-sequencing age. Nat Methods 11(4):361-362. https://doi.org/10.1038/nmeth.2890

41. Adzhubei IA, Schmidt S, Peshkin L, Ramensky VE, Gerasimova A, Bork P et al (2010) A method and server for predicting damaging missense mutations. Nat Methods 7(4):248-249. https://doi. org/10.1038/nmeth0410-248

42. Omasits U, Ahrens CH, Müller S, Wollscheid B (2013) Protter: interactive protein feature visualization and integration with experimental proteomic data. Bioinformatics 30(6):884-886. https://doi.org/10.1093/bioinformatics/btt607

43. Vaser R, Adusumalli S, Leng SN, Sikic M, Ng PC (2016) SIFT missense predictions for genomes. Nat Protoc 11(1):1-9. https:// doi.org/10.1038/nprot.2015.123

44. Consortium TU (2018) UniProt: a worldwide hub of protein knowledge. Nucleic Acids Res 47(D1):D506-D515. https://doi. org/10.1093/nar/gky1049

Publisher's note Springer Nature remains neutral with regard to jurisdictional claims in published maps and institutional affiliations. 